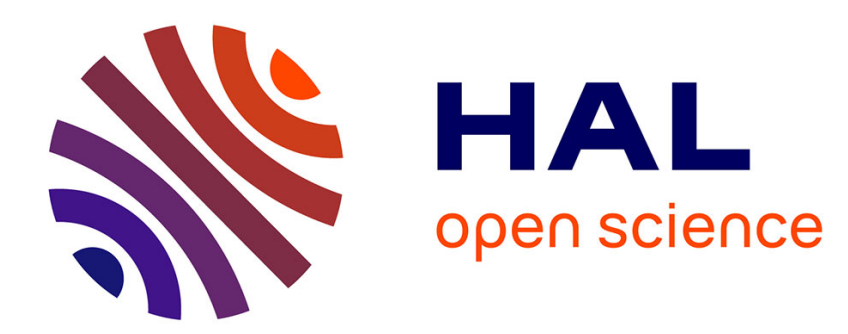

\title{
Displacement Derivatives in Shape Optimization of Thin Shells
}

Jan Sokolowski

\section{To cite this version:}

Jan Sokolowski. Displacement Derivatives in Shape Optimization of Thin Shells. [Research Report] RR-2995, INRIA. 1996, pp.21. inria-00073702

\section{HAL Id: inria-00073702 \\ https://hal.inria.fr/inria-00073702}

Submitted on 24 May 2006

HAL is a multi-disciplinary open access archive for the deposit and dissemination of scientific research documents, whether they are published or not. The documents may come from teaching and research institutions in France or abroad, or from public or private research centers.
L'archive ouverte pluridisciplinaire HAL, est destinée au dépôt et à la diffusion de documents scientifiques de niveau recherche, publiés ou non, émanant des établissements d'enseignement et de recherche français ou étrangers, des laboratoires publics ou privés. 


\section{Displacement Derivatives in Shape Optimization of Thin Shells}

Jan Sokołowski

\section{$N^{\circ} 2995$}

Octobre 1996

THÈME 4 



\title{
RINRIA
}

\section{Displacement Derivatives in Shape Optimization of Thin Shells}

\author{
Jan Sokołowski \\ Thème 4 - Simulation et optimisation \\ de systèmes complexes \\ Projet Numath
}

Rapport de recherche n2995 - Octobre 1996 - 21 pages

\begin{abstract}
In the present paper the framework for the shape sensitivity analysis of systems of equations defined on a surface in $\mathbb{R}^{3}$ is established. The model of thin shell presented in (Koiter, 1970) is considered. The formulation of the model in a reference domain has been chosen for our analysis. The shape gradients and shape Hessians of associated shape functionals are defined and evaluated using the so-called displacement derivatives.
\end{abstract}

Key-words: shape optimization, shape gradient, shape Hessian, thin shell, displacement derivative, surface shape functional

(Résumé : tsvp)

* The author is indebted to Michel Bernadou for reading the manuscript and many useful remarks.

Unité de recherche INRIA Lorraine

Technopôle de Nancy-Brabois, Campus scientifique,

615 rue de Jardin Botanique, BP 101, 54600 VILLERS LÈS NANCY (France)

Téléphone : (33) 83593030 - Télécopie : (33) 83278319

Antenne de Metz, technopôle de Metz 2000, 4 rue Marconi, 55070 METZ

Téléphone : (33) 87203500 - Télécopie : (33) 87763977 


\section{Dérivées de déplacement en optimisation de forme de coques minces}

Résumé : Dans cet article on établit le cadre de l'analyse de sensibilité par rapport à la forme de systèmes d'équations définis sur une surface de $\mathbb{R}^{3}$. On considère le modèle de coque mince présenté par (Koiter, 1970). La formulation du modèle dans un domaine de référence a été choisie pour notre analyse. Les gradients de forme et les Hessiens de forme associés aux fonctionnelles de forme ont été définis et évalués en utilisant la dérivée de déplacement.

Mots-clé : optimisation de forme, gradient de forme, Hessien de forme, coque mince, dérivée de déplacement, fonctionnelle de forme de surface 


\section{Introduction}

Shape optimization is quite indispensable when constructing industrial structures. For example plane and space shuttles have to satisfy in the same time very hard criteria on good mechanical behaviour and have to weight as less as possible.

For the last thirty years, there were many studies developed in this field of structural optimization. They combine the most recent results on mechanical formulation of the problems, on functional analysis of such problems and on control theory. For such results we can refer to e.g. (Duvaut, Lions, 1972) and to (Lions, 1968).

A structure is generally an assemblage between different parts like beams, plates, shells and three dimensional medium. Here we will restrict our attention to the general continuous formulation of such optimization problems for general thin shallow shells.

The geometry of a general thin shell can be characterized by two different mappings:

1. the mapping $\varphi$ which defines the middle surface $S$ of the shell as the image of the closure of a bounded domain $\mathcal{O}$ of the plane;

2. the mapping $e$ which defines the thickness of the shell at any point of the middle surface along the normal of this surface.

The shape optimization problem for such a shell consists in finding the geometry of the shell (middle surface and thickness) which minimizes a given functional (for example, the weight of the shell) and satisfies some constraints (for example, bounds on the thickness, on the strain energy, on the displacements).

We consider the following shape functional as an example,

$$
J(S)=\int_{S} \mathfrak{F}(x, \vec{u}(S)(x), \widetilde{e}(S)(x)) d \Gamma(x)
$$

where $\vec{u}(S)(x), x \in S$, is the displacement field of the shell.

We denote by $\mathfrak{J}(\varphi, e ; \vec{u})=J(S)$ the integral functional defined on the reference domain $\mathcal{O}$,

$$
\mathfrak{J}(\varphi, e ; \vec{u})=\int_{\mathcal{O}} \mathfrak{F}(\varphi(\xi), \mathcal{L} \cdot \vec{u}(\xi), e(\xi)) d S(\xi)
$$

with

$$
\begin{gathered}
\mathcal{L} \cdot \vec{u}(\xi) \equiv \vec{u}(S)(\varphi(\xi)), x=\varphi(\xi) \in S \\
e(\xi)=\widetilde{e}(S)(\varphi(\xi)) \quad \xi \in \mathcal{O}
\end{gathered}
$$

RR n 2995 
where $\vec{u}(\xi)=\operatorname{col}\left(u_{1}(\xi), u_{2}(\xi), u_{3}(\xi)\right)$ denotes covariant components of the displacement field $\vec{u}(S) \circ \varphi$ of the middle surface $\bar{S}$, and linear mapping $\mathcal{L}$ is defined in terms of contravariant basis on $S$, i.e. $\mathcal{L} \cdot \vec{u}=u_{i} \vec{a}^{i}$.

Computational algorithms usually require that we can compute the derivatives of shape functionals with respect to the geometry, i.e., with respect to $S=\varphi(\mathcal{O})$ and $e$. This is a difficult problem since in general shape functionals depend on $S$ and $e$ not only explicitely but also implicitly through the dependence of the displacement field $\vec{u}(S)$ on $S$ and $e$. To circumvent this difficulty we make use of the classical adjoint state method.

We use the shell model presented in (Koiter, 1970). A detailed discussion related to numerical analysis of such problems can be found in (Bernadou, Ciarlet, 1976), (Bernadou, Boisserie, 1982) or (Bernadou, 1995).

\section{Shell model}

The following hypothesis are assumed to be satisfied.

1. the shell is clamped on its boundary $\partial S$;

2. the shell is loaded by a distribution of forces whose resultant has density $\vec{p}$ on $S$,

3. the shell is elastic, homogenous and isotropic.

Moreover, according to (Koiter, 1970), we assume that

4. the normals to the middle surface remain normals to the deformed middle surface;

5. the stresses are approximatively plane and parallel to the tangent plane to the middle surface.

Under the assumptions (1) to (5) above, the problem takes the following variational form.

Find $\vec{u}=\left(u_{1}, u_{2}, u_{3}\right) \in \overrightarrow{\mathcal{H}}=\left(H_{0}^{1}(\mathcal{O})\right)^{2} \times H_{0}^{2}(\mathcal{O})$ such that

$$
a(\vec{u}, \vec{v})=f(\vec{v}) \quad \forall \vec{v} \in \mathcal{H},
$$

where

$$
a(\vec{u}, \vec{v})=\int_{\mathcal{O}} e E^{\alpha \beta \lambda \iota}\left\{\gamma_{\alpha \beta}(\vec{u}) \gamma_{\lambda_{\iota}}(\vec{v})+\frac{e^{2}}{12} \rho_{\alpha \beta}(\vec{u}) \rho_{\lambda \iota}(\vec{v})\right\} d S,
$$




$$
\begin{gathered}
f(\vec{v})=\int_{\mathcal{O}} \vec{p} \cdot \vec{v} d S \\
E^{\alpha \beta \lambda \iota}=\frac{E}{2(1+\mu)}\left[a^{\alpha \lambda} a^{\beta \iota}+a^{\alpha \iota} a^{\beta \lambda}+\frac{2 \mu}{1-\mu} a^{\alpha \beta} a^{\lambda \iota}\right] \\
d S=\sqrt{a} d \xi^{1} d \xi^{2} \\
E=\text { Young modulus } ; \quad \nu=\text { Poisson's coefficient. } \\
\gamma_{\alpha \beta}(\vec{u})=\frac{1}{2}\left(u_{\alpha \mid \beta}+u_{\beta \mid \alpha}\right)-b_{\alpha \beta} u_{3} \\
\bar{\rho}_{t \alpha \beta}\left(\vec{u}_{t}\right)=u_{t 3 \mid \alpha \beta}-b_{t \alpha}^{\lambda} b_{t \lambda \beta} u_{t 3}+b_{t \alpha \mid \beta}^{\lambda} u_{t \lambda}+b_{t \alpha}^{\lambda} u_{t \lambda \mid \beta}+b_{t \beta}^{\lambda} u_{t \lambda \mid \alpha}
\end{gathered}
$$

These expressions can be simplified by taking into account deformations of general thin shallow shells. This allows to keep $\gamma_{\alpha \beta}$ unchanged and to replace $\bar{\rho}_{\alpha \beta}(\vec{u})$ by

$$
\rho_{\alpha \beta}(\vec{u})=u_{3 \mid \alpha \beta}
$$

Theorem 2.1 Problem (2.1) has a unique solution.

The proof of Theorem 2.1 is given in (Bernadou, Ciarlet, 1976), we refer the reader also to (Bernadou, Ciarlet and Miara, 1994) for a more simple proof.

Theorem 2.2 Problem (2.1) with $\bar{\rho}_{\alpha \beta}(\vec{u})$ replaced by $\rho_{\alpha \beta}(\vec{u})$ (see (2.7)) has a unique solution.

The proof of Theorem 2.2 is given in (Bernadou, Lalane, 1986) or in (Bernadou, 1994 or 1995).

\section{Displacement derivatives}

Basic assumption we make in this section is that the shape functional $J(S)=$ $\mathfrak{J}(\varphi, e ; \vec{u})$ under consideration depends only on $S=\varphi(\mathcal{O})$ and $\widetilde{e}$ and is independent of the parametrization $\varphi$ for a fixed surface $S$ and the given reference domain $\mathcal{O}$.

Under this assumption, by an application of the Hadamard formula (see section 4 for details), we can obtain the form of shape gradient of the shape functional $J(S)=\mathfrak{J}(\varphi, e ; \vec{u})$. In particular, we can use displacement derivatives of solutions to shell equations to evaluate the Eulerian derivative $d J(S ; V)$ in the direction $V$.

RR n 2995 
Jan Sokolowski

\section{Notation}

Let $\mathcal{O}$ be a bounded domain of a plane $\mathbb{R}^{2}$ with boundary $\Gamma=\partial \mathcal{O}$. We assume that the middle surface $\bar{S}$ of the shell is the image of the set $\overline{\mathcal{O}}$ under a regular mapping $\varphi$, i.e. $\bar{S}=\varphi(\overline{\mathcal{O}})$,

$$
\varphi:\left(\xi^{1}, \xi^{2}\right) \in \overline{\mathcal{O}} \subset \mathbb{R}^{2} \mapsto \varphi\left(\xi^{1}, \xi^{2}\right) \in \bar{S} \subset \mathbb{R}^{3}
$$

We define two local bases in $\bar{S}$ :

1. the covariant basis $\left(\vec{a}_{i}, i=1,2,3\right)$

$$
\vec{a}_{\alpha}=\varphi_{, \alpha}=\frac{\partial \varphi}{\partial \xi^{\alpha}}, \quad \vec{a}_{3}=\frac{\vec{a}_{1} \times \vec{a}_{2}}{\left\|\vec{a}_{1} \times \vec{a}_{2}\right\|_{\mathbb{R}^{3}}} ;
$$

2. the contravariant basis $\left(\vec{a}^{i}, i=1,2,3\right)$

$$
\vec{a}^{\beta} \cdot \vec{a}_{\alpha}=\delta_{\alpha \beta} \quad ; \quad \vec{a}^{3}=\vec{a}_{3} .
$$

From now on small greek indices take values 1 and 2 while small latin indices take values 1,2 and 3 . We use Einstein's summation convention for repeated indices at higher and lower positions.

To the covariant and contravariant bases we assign the first $\left(a_{\alpha \beta}, a^{\alpha \beta}\right)$ and the second $\left(b_{\alpha \beta}, b_{\alpha}^{\beta}, b^{\alpha \beta}\right)$ fundamental forms of the middle surface (note that $b_{\alpha}^{\cdot \beta}=b_{\cdot \alpha}^{\beta}=$ $\left.b_{\alpha}^{\beta}\right)$, respectively,

$$
\begin{gathered}
a_{\alpha \beta}=\vec{a}_{\alpha} \cdot \vec{a}_{\beta} ; \quad a^{\alpha \beta}=\vec{a}^{\alpha} \cdot \vec{a}^{\beta} ; \quad a=\operatorname{det}\left(a_{\alpha \beta}\right) \\
b_{\alpha \beta}=\vec{a}_{3} \cdot \vec{a}_{\alpha, \beta} ; \quad b_{\alpha}^{\beta}=a^{\beta \lambda} b_{\lambda \alpha} ; \quad b^{\alpha \beta}=a^{\alpha \lambda} b_{\lambda}^{\beta} .
\end{gathered}
$$

It is also convenient to introduce the covariant derivatives

$$
u_{\alpha \mid \beta}=u_{\alpha, \beta}-\Gamma_{\alpha \beta}^{\lambda} u_{\lambda} ; \quad u_{3 \mid \lambda}=u_{3, \alpha} ; \quad u_{3 \mid \alpha \beta}=u_{3, \alpha \beta}-\Gamma_{\alpha \beta}^{\lambda} u_{3, \lambda},
$$

where the Christoffel symbols are given as

$$
\Gamma_{\alpha \beta}^{\lambda}=\vec{a}^{\lambda} \cdot \vec{a}_{\alpha, \beta}=\Gamma_{\beta \alpha}^{\lambda} .
$$

Subsequently, the same notation will be used for the surface $S_{t}$.

The thickness of the shell can be defined as a regular mapping

$$
e:\left(\xi^{1}, \xi^{2}\right) \in \overline{\mathcal{O}} \subset \mathbb{R}^{2} \mapsto e\left(\xi_{1}, \xi_{2}\right) \in\{x \in \mathbb{R}: x>0\} .
$$

Then the shell $\mathcal{S}$ is the set

$$
\begin{aligned}
\mathcal{S}= & \left\{M \in \mathbb{R}^{3}: \overrightarrow{O M}=\varphi\left(\xi^{1}, \xi^{2}\right)+\xi^{3} \vec{a}_{3}\left(\xi^{1}, \xi^{2}\right),\left(\xi^{1}, \xi^{2}\right) \in \overline{\mathcal{O}}\right. \\
& \left.-\frac{1}{2} e\left(\xi^{1}, \xi^{2}\right) \leq \xi^{3} \leq \frac{1}{2} e\left(\xi^{1}, \xi^{2}\right)\right\}
\end{aligned}
$$




\section{The family of surfaces $S_{t}$}

By $t$ we denote a real parameter which belongs to $[0, \delta], \delta>0$. Let $S_{t}$ be a family of surfaces defined as the images of the reference plane domain $\mathcal{O}$ under regular mappings $\varphi_{t}$ :

$$
\varphi_{t}:\left(\xi^{1}, \xi^{2}\right) \in \overline{\mathcal{O}} \subset \mathbb{R}^{2} \mapsto \varphi_{t}\left(\xi^{1}, \xi^{2}\right) \in \overline{S_{t}} \subset \mathbb{R}^{3}
$$

For $t=0$ we recover the original surface $S$, i.e.,

$$
\varphi_{0}=\varphi, \quad \text { and } \quad S_{0}=S \text {. }
$$

It follows that

$$
\frac{\partial \varphi_{t}}{\partial t}=c_{t}^{\alpha} \vec{a}_{t \alpha}+w_{t} \vec{a}_{t 3}
$$

and for convected parametrizations, i.e. when $c_{t}^{\alpha}=0$ we have

$$
\frac{\partial \varphi_{t}}{\partial t}=w_{t} \vec{a}_{t 3}
$$

where $w_{t}$ is the normal speed of the surface $S_{t}$, and $\vec{a}_{t 3}$ is the unit normal vector to $S_{t}$.

For any parametrization of the surface $S_{t}$ we have

$$
\frac{\partial \varphi_{t}}{\partial t}=c_{t}^{\alpha} \vec{a}_{t \alpha}+\frac{\delta \varphi_{t}}{\delta t}
$$

where $\frac{\delta}{\delta t}$ denotes the displacement derivative defined below (Definition 3.1).

Definition 3.1 Let $z_{t}: \mathcal{O} \mapsto \mathbb{R}, t \in[0, \delta]$ be a family of functions and let $\varphi_{t}$, $t \in[0, \delta)$, be a family of regular mappings, $\bar{S}_{t}=\varphi_{t}(\overline{\mathcal{O}})$. The displacement derivative $\frac{\delta z_{t}}{\delta t}$ for this family of functions is defined by

$$
\frac{\delta z_{t}}{\delta t}=\frac{\partial z_{t}}{\partial t}-z_{t, \alpha} g^{\alpha \beta} \varphi_{t, \beta} \cdot \frac{\partial \varphi_{t}}{\partial t}
$$

Remark 3.2 Using the material derivative method a mapping $T_{t}=T_{t}(V): \mathbb{R}^{3} \mapsto$ $\mathbb{R}^{3}$ is constructed where $V$ is a given vector field. The mapping, in the case of thin shell, assigns the surface $S_{t}$ to the reference surface $S$, i.e.,

$$
S_{t}=T_{t}(S)
$$


For

$$
\varphi_{t}=T_{t} \circ \varphi=T_{t}(V) \circ \varphi, \quad t \in[0, \delta)
$$

we have

$$
\frac{\partial \varphi_{t}}{\partial t}=\left[\frac{\partial T_{t}}{\partial t} \circ T_{t}^{-1}\right] \circ \varphi=V(t) \circ \varphi_{t}=V\left(t, \varphi_{t}\right)
$$

therefore

$$
\frac{\delta z_{t}}{\delta t}=\frac{\partial z_{t}}{\partial t}-z_{t, \alpha} g^{\alpha \beta} \varphi_{t, \beta} \cdot V\left(t, \varphi_{t}\right)
$$

It is easily seen that the displacement derivative of $\varphi_{t}$ takes the form

$$
\frac{\delta \varphi_{t}}{\delta t}=\left\langle V\left(t, \varphi_{t}\right), \vec{a}_{3 t}\right\rangle_{\mathbb{R}^{3}} \vec{a}_{3 t}=w_{t} \vec{a}_{3 t} .
$$

Now we determine the displacement derivative of the restriction of a function to $S$. Let $\psi:[0, \delta) \times D \mapsto \mathbb{R}$. Denoting $u_{t}=\psi\left(t, \varphi_{t}\right)$, we get

$$
\begin{aligned}
& \frac{\partial u_{t}}{\partial t}(\xi)=\frac{\partial \psi}{\partial t}\left(t, \varphi_{t}(\xi)\right)+\left\langle\nabla_{x} \psi\left(t, \varphi_{t}(\xi)\right), V\left(t, \varphi_{t}(\xi)\right)\right\rangle_{\mathbb{R}^{3}}, \\
& \frac{\delta u_{t}}{\delta t}(\xi)=\frac{\partial \psi}{\partial t}(t, \xi)+\left\langle\nabla_{x} \psi\left(t, \varphi_{t}(\xi)\right), \vec{a}_{t 3}\right\rangle_{\mathbb{R}^{3}} w_{t}(\xi) .
\end{aligned}
$$

If $z^{\prime}(S ; V)$ is the boundary shape derivative of $z\left(S_{t}\right)=\psi_{\mid S_{t}}$ in the direction of a vector field $V(t)=\frac{\partial T_{t}}{\partial t} \circ T_{t}^{-1}$, it follows that the following relation is obtained

$$
{\frac{\delta u_{t}}{\delta t}}_{\mid t=0}(\xi)=z^{\prime}(S ; V)(\varphi(\xi))
$$

For $t>0$ we use the following notation,

$$
\frac{\delta u_{t}}{\delta t}(\xi)=z^{\prime}\left(S_{t} ; V(t)\right)\left(\varphi_{t}(\xi)\right) \text {. }
$$

It is clear that if $\frac{\partial \psi}{\partial t}=\frac{\partial \psi}{\partial n}=0$ on $S$ and $u_{t}=\psi\left(t, \varphi_{t}\right)$, then $\frac{\delta u_{t}}{\delta t}=0$ for $t=0$.

Remark 3.3 Given mappings $\varphi_{t}, \varphi$, we can define the mapping

$$
T_{t}: S \mapsto S_{t}
$$

of the form $T_{t}=\varphi_{t} \circ \varphi^{-1}$, and the vector field

$$
V(t, x)=\left[\frac{\partial T_{t}}{\partial t} \circ T_{t}^{-1}\right](x)=\left[\frac{\partial \varphi_{t}}{\partial t} \circ \varphi_{t}^{-1}\right](x) \quad x \in S_{t} .
$$


The normal and tangent components of the field $V$ on $S$ are given by

$$
\begin{aligned}
& w(t, x)=\langle V(t, x), n(t, x)\rangle_{\mathbb{R}^{3}} \quad x \in S_{t}, \\
& V_{\tau}(t, x)=V(t, x)-w(t, x) n(t, x), \\
& w_{t}(\xi)=w\left(t, \varphi_{t}(\xi)\right), \\
& n(t, x)=\vec{a}_{t 3}\left(\varphi_{t}^{-1}(x)\right) \quad x \in S_{t} .
\end{aligned}
$$

Therefore, without any loss of generality we assume that there is given a mapping $\varphi$ and an admissible vector field $V$ such that $\varphi_{t}=T_{t}(V) \circ \varphi$.

A tangent vector on $\partial S_{t}=\left\{x \in \mathbb{R}^{3} \mid x=\varphi(\xi) \quad \xi \in \partial \mathcal{O}\right\}$ is denoted by $\vec{b}_{t}(\xi)$; $\vec{l}_{t}(\xi)=\Sigma_{i=1}^{2} \alpha_{t}^{i}(\xi) \vec{a}_{i t}(\xi)$ is the unit vector normal to $\partial S_{t}$ such that $\left\langle\vec{l}_{t}(\xi), \vec{b}_{t}(\xi)\right\rangle_{\mathbb{R}^{3}}=0$. For $t=0$ we denote $\vec{b}(\xi)=\vec{b}_{0}(\xi), \vec{l}(\xi)=\vec{l}_{0}(\xi)$, respectively; $\vec{l}(t, x)=\vec{l}_{t}\left(\varphi_{t}^{-1}(\xi)\right)$ for $x=\varphi_{t}(\xi) \in \partial S_{t}, \vec{b}(t, x)$ is defined in the same way for $x \in \partial S_{t}$.

\section{Derivatives of shape functionals}

We recall here the basic notions of the shape calculus which are used in the paper. For further results on the material derivative method we refer the reader to (Sokolowski and Zolesio, 1992). Shape optimization problems in solid mechanics are considered in (Khludnev and Sokolowski, to appear)

Suppose we are given an open set $D$ in $\mathbb{R}^{N}$, a measurable subset $\Omega$ of $D$, an admissible vector field $V \in C\left(0, \varepsilon ; C^{k}\left(\bar{D} ; \mathbb{R}^{N}\right)\right), k \geq 1$, and an associated transformation $T_{t}(V)$ from $\bar{D}$ onto $\bar{D}$.

Let $J(\Omega)$ be a well defined functional for any measurable subset $\Omega$ of $D$. Assume that $\Omega_{t}=T_{t}(V)(\Omega), t \in[0, \delta)$, is a family of deformations of $\Omega$. The set $\Omega_{t}$ is a measurable subset of $D$ for any $t \in[0, \delta)$.

Definition 4.1 For an admissible vector field $V \in C\left(0, \varepsilon ; C^{k}\left(\bar{D} ; \mathbb{R}^{N}\right)\right)$, the Eulerian derivative of the domain functional $J(\Omega)$ at $\Omega$ in the direction of $V$ is the limit

$$
d J(\Omega ; V)=\lim _{t \downarrow 0}\left(J\left(\Omega_{t}\right)-J(\Omega)\right) / t
$$

where

$$
\Omega_{t}=T_{t}(V)(\Omega)
$$

Definition 4.2 A functional $J(\Omega)$ is shape differentiable (or simply differentiable) at $\Omega$ if

RR n2995 
1. there exists the Eulerian derivative $d J(\Omega ; V)$ for all directions $V$,

2. the mapping $V \mapsto d J(\Omega ; V)$ is a linear and continuous mapping from $C(0, \varepsilon$; $\left.C^{k}\left(D ; \mathbb{R}^{N}\right)\right)$ into $\mathbb{R}$.

Gradients of shape differentiable functionals can be characterized as follows.

Theorem 4.3 Let $J(\Omega)$ be a shape differentiable functional at every domain $\Omega$ of class $C^{k}, \Omega \subset D$. Assume that $\Omega \subset D$ is a domain with the boundary of class $C^{k-1}$. There exists a scalar distribution

$$
g(\Gamma) \in \mathcal{D}^{-k}(\Gamma)
$$

such that the gradient of the functional $J$ at $\Omega, G(\Omega) \in \mathcal{D}^{-k}\left(\Omega ; \mathbb{R}^{N}\right)$, with $\operatorname{spt} G(\Omega) \in$ $\Gamma$, is given by

$$
G(\Omega)={ }^{*} \gamma_{\Gamma}(g \cdot n)
$$

where $\gamma_{\Gamma} \in \mathcal{L}\left(\mathcal{D}\left(\bar{D} ; \mathbb{R}^{N}\right), \mathcal{D}\left(\Gamma ; \mathbb{R}^{N}\right)\right)$ is the trace operator and ${ }^{*} \gamma_{\Gamma}$ is the transpose of $\gamma_{\Gamma}, n$ is a unit normal vector on $\Gamma$ directed into the exterior of $\Omega$.

From (4.2) it follows that

$$
d J(\Omega ; V)=\langle g, V \cdot n\rangle_{\mathcal{D}^{-k}(\Gamma) \times \mathcal{D}^{k}(\Gamma)},
$$

where $V \cdot n=\langle V(0, x), n(x)\rangle_{\mathbb{R}^{N}}, x \in \Gamma$.

In general, $g=g(\Omega) \in \mathcal{D}^{-k}(\Gamma)$. However, for some classes of shape functionals it can be assumed that $g(\Omega)$ is an integrable function on $\Gamma$ and then

$$
d J(\Omega ; V)=\int_{\Gamma} g(x)\langle V(0, x), n(x)\rangle_{\mathbb{R}^{N}} d \Gamma
$$

and we denote

$$
\mathfrak{D} J(\Omega ; V(0))=\int_{\Gamma} g(x)\langle V(0, x), n(x)\rangle_{\mathbb{R}^{N}} d \Gamma .
$$

Let $\bar{S}=\varphi(\overline{\mathcal{O}})$, and let $J(S)$ be a given differentiable shape functional. For any vector field $V$ such that $\operatorname{spt} V(0) \cap \partial S=\emptyset$, the Eulerian derivative $d J(S ; V)=$ $\left.\lim _{t \downarrow 0}\left(J\left(S_{t}\right)-J(S)\right)\right) / t$ of the shape functional takes the following form.

Corollary 4.4 There exists a distribution $\mathcal{G}_{S} \in \mathcal{D}^{-k}(S)$ such that

$$
d J(S ; V)=\left\langle\mathcal{G}_{S}, w\right\rangle_{\mathcal{D}^{-k}(S) \times \mathcal{D}^{k}(S)},
$$

where, $w$ is the normal speed of the surface $S_{t}$ at $t=0$, i.e. $w(x)=w(\varphi(\xi))=$ $\left\langle V(0, \varphi(\xi)), \vec{a}_{3}(\xi)\right\rangle_{\mathbb{R}^{3}}$, for $x=\varphi(\xi) \in S$ and $\xi=\varphi^{-1}(x) \in \mathcal{O}$. 


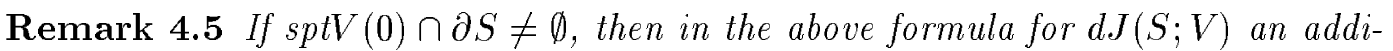
tional term related to the boundary $\partial S$ may appear. The term takes the form

$$
\left\langle\overrightarrow{\mathcal{G}}_{\partial S}, V_{\ell}(0)\right\rangle_{\mathcal{D}^{-k}\left(\partial S ; \mathbb{R}^{3}\right) \times \mathcal{D}^{k}\left(\partial S ; \mathbb{R}^{3}\right)}
$$

where $V_{\ell}(0)$ is a component of tangent vector field $\gamma_{\partial S} V_{\tau}(0)$ orthogonal to the tangent vector $\vec{b}$ on $C^{1}$ curve $\partial S$, i.e. $V_{\ell}(0, \varphi(\xi))=\langle V(0, \varphi(\xi)), \vec{l}(\xi)\rangle_{\mathbb{R}^{3}} \vec{l}(\xi)$ for $\xi \in \partial \mathcal{O}$. $\gamma_{\partial S} V_{\tau}(0)$ denotes the trace of $V_{\tau}(0)=V-\langle V, n\rangle_{\mathbb{R}^{3}} n$ on $\partial S$.

We recall the formulae for the derivatives of integrals. Given a family of shape differentiable functions $y\left(\Omega_{t}\right), y\left(\Gamma_{t}\right), \Omega_{t}=T_{t}(\Omega)$, then

$$
\begin{aligned}
& \frac{d}{d t}\left[\int_{\Omega_{t}} y\left(\Omega_{t}\right) d x\right]_{\mid t=0}=\int_{\Omega} y^{\prime}(\Omega ; V) d x+\int_{\partial \Omega} y(\Omega)\langle V(0), n\rangle_{\mathbb{R}^{N}} d \Gamma \\
& \frac{d}{d t}\left[\int_{\Gamma_{t}} z\left(\Gamma_{t}\right) d x\right]_{\mid t=0}=\int_{\Gamma} z^{\prime}(\Gamma ; V)-2 \kappa z(\Gamma)\langle V(0), n\rangle_{\mathbb{R}^{N}} d \Gamma
\end{aligned}
$$

where $\kappa=-\frac{1}{2} \operatorname{div}_{\Gamma} n$ is the mean curvature on $\partial \Omega$.

Let us consider a surface integral $J(S)=\int_{S} G d \Gamma$. For $S=\varphi(\mathcal{O}) \subset \mathbb{R}^{3}$ it follows that $J(S)=\int_{\mathcal{O}} G \circ \varphi d S, d S=a^{\frac{1}{2}} d \xi$, where $a=\operatorname{det}\left[a_{\alpha, \beta}\right]$.

Given vector field $V$ and the transformation $T_{t}=T_{t}(V): S \mapsto S_{t}$, we denote $\varphi_{t}=T_{t} \circ \varphi: \mathcal{O} \mapsto S_{t}, V(t, x)=\left[\frac{\partial T_{t}}{\partial t} \circ T_{t}^{-1}\right](x)$ for $x \in S_{t}$. Consider the shape functional defined on $S_{t}$,

$$
\begin{aligned}
J\left(S_{t}\right) & =\int_{S_{t}} F(t) d \Gamma=\int_{S_{t}} z\left(S_{t}\right) d \Gamma \\
& =j(t)=\int_{\mathcal{O}} F_{t} d S_{t}
\end{aligned}
$$

where $F: \mathbb{R} \times \mathbb{R}^{3} \mapsto \mathbb{R}$ is a given sufficiently smooth function and the following notation is used,

$$
\begin{aligned}
& z\left(S_{t}\right)=F(t, \cdot)_{\mid S_{t}}, \\
& F_{t}(\xi)=F\left(t, \varphi_{t}(\xi)\right) \quad \xi \in \mathcal{O}, \\
& d S_{t}=\operatorname{det}\left(\left[D T_{t}\right] \circ \varphi\right)\left\|\left[{ }^{*} D T_{t}^{-1} \circ \varphi\right] \cdot \vec{a}_{t 3}\right\|_{\mathbb{R}^{3}} d S .
\end{aligned}
$$

We are going to evaluate the second order shape derivative of the shape functional $J\left(S_{t}\right)$ in the direction of vector fields $V, W$. For the first order shape derivative we 
have the following representations

$$
\begin{aligned}
d J\left(S_{t} ; V(t)\right) & =\int_{S_{t}} z^{\prime}\left(S_{t} ; V(t)\right)-2 \kappa(t) z\left(S_{t}\right)\langle V(t), n(t)\rangle_{\mathbb{R}^{3}} d \Gamma_{t} \\
& =\int_{S_{t}} \frac{\partial F}{\partial t}(t)+\left(\frac{\partial F}{\partial n}(t)-2 \kappa(t) F(t)\right)\langle V(t), n(t)\rangle_{\mathbb{R}^{3}} d \Gamma_{t} \\
& =\frac{d j}{d t}(t)=\int_{\mathcal{O}}\left[\frac{\delta F_{t}}{\delta t}-2 \kappa_{t} F_{t} w_{t}\right] d S_{t}
\end{aligned}
$$

where we denote

$$
\begin{aligned}
& \kappa(t, x) \text { is the mean curvature at } x \in S_{t}, \\
& n(t)=\vec{a}_{t 3} \circ \varphi_{t}^{-1} \text { the normal vector at } x=\varphi_{t}^{-1}(\xi) \in S_{t}, \xi \in \mathcal{O}, \\
& \frac{\partial F}{\partial n}(t)=\langle\nabla F(t), n(t)\rangle_{\mathbb{R}^{3}}, \\
& \kappa_{t}(\xi)=\kappa\left(t, \varphi_{t}(\xi)\right), \\
& w_{t}(\xi)=\left\langle\frac{\delta \varphi_{t}}{\delta t}(\xi), \vec{a}_{t 3}(\xi)\right\rangle_{\mathbb{R}^{3}}, \xi \in \mathcal{O} .
\end{aligned}
$$

Remark 4.6 In general, we have the following formula for derivative of a surface integral, we refer the reader to (Sokolowski, Zolesio, 1992) for the proof.

Let $\Sigma$ be a connected surface contained in $\Gamma$ with $C^{2}$ boundary $\partial \Sigma, \vec{l}$ denote the unit normal vector to $\partial \Sigma$ that is perpendicular to the surface normal $n$ to $\Gamma$ and directed into the exterior of $\Sigma$. For $\Sigma_{t}=T_{t}(\Sigma) \subset \Gamma_{t}$ it follows that,

$$
\begin{aligned}
& \frac{d}{d t}\left[\int_{\Sigma_{t}} z\left(\Gamma_{t}\right) d \Gamma_{t}\right]_{\mid t=0}=\frac{d}{d t}\left[\int_{\Sigma} z\left(\Gamma_{t}\right) \circ T_{t}(V) \omega(t) d \Gamma\right]_{\mid t=0} \\
= & \int_{\Sigma} \dot{z}(\Gamma ; V)+\dot{\omega}(\Gamma ; V) z(\Gamma) d \Gamma \\
= & \int_{\Sigma} z^{\prime}(\Gamma ; V)+\nabla_{\Gamma} z(\Gamma) \cdot V_{\tau}(0)-2 \kappa z(\Gamma) V(0) \cdot n+z(\Gamma) \operatorname{div}_{\Gamma} V_{\tau}(0) d \Gamma \\
= & \int_{\Sigma} z^{\prime}(\Gamma ; V)-2 \kappa z(\Gamma) V(0) \cdot n d \Gamma+\int_{\Sigma} \operatorname{div}_{\Gamma}\left(z(\Gamma) V_{\tau}(0)\right) d \Gamma \\
= & \int_{\Sigma} z^{\prime}(\Gamma ; V)-2 \kappa z(\Gamma) V(0) \cdot n d \Gamma+\int_{\partial \Sigma} z(\Gamma)\left\langle V_{\tau}(0), \vec{l}_{\mathbb{R}^{3}} d l,\right.
\end{aligned}
$$

where $\omega(t)=\gamma(t)\left\|^{*} D T_{t}^{-1} \cdot n(t)\right\|_{\mathbb{R}^{3}}$ and $\dot{\omega}=-2 \kappa V(0) \cdot n+\operatorname{div}_{\Gamma} V_{\tau}(0)$ in the notation of (Sokolowski, Zolesio, 1992). 
To differentiate the shape functional

$$
I(S)=\mathfrak{D} J(S ; V(0))
$$

in the direction of a vector field $W$ we need the following notation, $r \in(-\epsilon, \epsilon)$ is a parameter,

$$
\begin{aligned}
& S_{r}=T_{r}(S), \text { where } T_{r}=T_{r}(W): S \mapsto S_{r}, \\
& V(r)=V\left(r, \varphi_{r}(\xi)\right), \\
& \varphi_{r}=T_{r}(W) \circ \varphi, \\
& w_{r}(\xi)=\left\langle\frac{\delta \varphi_{r}}{\delta r}(\xi), \vec{a}_{r 3}(\xi)\right\rangle_{\mathbb{R}^{3}}, \\
& F_{r}(\xi)=F\left(r, \varphi_{r}(\xi)\right), \\
& \kappa_{r}(\xi)=\kappa\left(r, \varphi_{r}(\xi)\right), \\
& \frac{\delta F_{r}}{\delta t}(\xi)=\frac{\partial F}{\partial t}\left(r, \varphi_{r}(\xi)\right)+\left\langle\nabla_{x} F\left(r, \varphi_{r}(\xi)\right), \vec{a}_{r 3}(\xi)\right\rangle_{\mathbb{R}^{3}}, \xi \in \mathcal{O} .
\end{aligned}
$$

With the notation we have

$$
\begin{aligned}
I\left(S_{r}\right) & =d J\left(S_{r} ; V(r)\right) \\
& =\int_{S_{r}} z^{\prime}\left(S_{r} ; V(r)\right)-2 \kappa(r) z\left(S_{r}\right)\langle V(r), n(r)\rangle_{\mathbb{R}^{3}} d \Gamma \\
& =\int_{S_{r}} \frac{\partial F}{\partial t}(r)+\left(\frac{\partial F}{\partial n}(r)-2 \kappa(r) F(r)\right)\langle V(r), n(r)\rangle_{\mathbb{R}^{3}} d \Gamma \\
& =\frac{d j}{d t}(r)=\int_{\mathcal{O}}\left[\frac{\delta F_{r}}{\delta t}-2 \kappa_{r} F_{r} w_{r}\right] d S_{r}
\end{aligned}
$$

For $u\left(S_{r}\right)=z^{\prime}\left(S_{r} ; V(r)\right)-2 \kappa(r) z\left(S_{r}\right)\langle V(r), n(r)\rangle_{\mathbb{R}^{3}}$ and $u_{r}=u\left(S_{r}\right) \circ \varphi_{r}=\frac{\delta F_{r}}{\delta t}-$ $2 \kappa_{r} F_{r} w_{r}$ it follows that

$$
\begin{aligned}
& \mathfrak{D} I(S ; W(0))=\mathfrak{D}^{2} J(S ; V(0), W(0)) \\
= & \int_{S} u^{\prime}(S ; W)-2 \kappa u(S)\langle W(0), n\rangle_{\mathbb{R}^{3}} d \Gamma \\
= & {\left[\frac{d}{d r} \frac{d j}{d t}(r)\right]_{\mid r=0}=\left.\int_{\mathcal{O}} \frac{\delta u_{r}}{\delta r}\right|_{\mid r=0}-2\left(\kappa_{r} u_{r} w_{r}\right)_{\mid r=0} d S }
\end{aligned}
$$

In the present paper the second order derivatives of shape functionals are evaluated for the fields $W=V=\frac{\partial \varphi_{t}}{\partial t} \circ \varphi_{t}^{-1}$. 
The second order derivative $d^{2} J(\Omega ; V, W)$ of the shape functional $J(\Omega)$ in the direction of vector fields $V, W$ is defined as follows

$$
d^{2} J(\Omega ; V, W)=\lim _{s \downarrow 0} \frac{1}{s}\left[d J\left(\Omega_{s} ; V(s)\right)-d J(\Omega ; V)\right],
$$

where $\Omega_{s}=T_{s}(W)(\Omega), V(s)=V(s, x)$ with $x=T_{s}(W)(X)$ for $X \in \Omega$.

It can be shown, that we have the following representation of the second order shape derivative, if the shape derivative exists,

$$
\begin{aligned}
& d^{2} J(\Omega ; V, W)=\mathfrak{d}^{2} J(\Omega ; V(0), W(0))+\mathfrak{D} J(\Omega ; \dot{V}(0)), \\
& \mathfrak{D} J(\Omega ; \dot{V}(0))=\mathfrak{D} J\left(\Omega ; \partial_{t} V(0)\right)+\mathfrak{D} J(\Omega ;[D V \cdot W](0))
\end{aligned}
$$

where $\dot{V}(0)$ denotes the material derivative of the velocity field $V$ in the direction of the field $W$,

$$
\dot{V}(0, \cdot)=\frac{\partial V}{\partial t}(0, \cdot)+[D V \cdot W](0, \cdot)=\partial_{t} V(0)+[D V \cdot W](0) .
$$

Definition 4.7 The linear operator $\mathcal{D}\left(D ; \mathbb{R}^{N}\right) \mapsto \mathcal{D}^{\prime}\left(D ; \mathbb{R}^{N}\right)$ associated with the symmetric bilinear form $\mathfrak{d}^{2} J(\Omega ; \cdot, \cdot)$ is called the Shape Hessian.

Whenever it exists the Shape Hessian is a symmetric operator. The form of the Shape Hessian can be identified from the second order shape derivative $d^{2} J(\Omega ; V, W)$ by taking the vector fields $V$ such that $\dot{V}(0)=0$. Therefore, at least at the first stage, the material derivatives can be used in order to evaluate the second order shape derivative of a specific shape functional.

On the other, the second order Eulerian derivative

$$
\mathfrak{D}^{2} J(\Omega ; V(0), W(0)) \equiv \mathfrak{d}^{2} J(\Omega ; V(0), W(0))+\mathfrak{D} J(\Omega ;[D V \cdot W](0))
$$

can be evaluated by taking the shape derivative of the shape functional $I(\Omega)=$ $\mathfrak{D} J(\Omega ; V(0))$ in the direction of a vector field $W$, we refer the reader to eg. (Delfour, Zolesio, 1991) for related results. In particular, our definition of the Shape Hessian is stricly different from the definition given in (Delfour, Zolesio, 1991), where the nonsymmetric shape Hessian is introduced. It is also a difficult task to evaluate the symmetric part of the shape Hessian directly from the Eulerian derivative $\mathfrak{D}^{2} J(\Omega ; V(0), W(0))$ as it was observed in (Novruzi, Roche, 1994) where the relation similar to (4.6) was established for a specific problem of shape optimization. The Shape Hessian is required for the applications to the Newton method (Novruzi, Roche, 1994) as well as to the stability analysis of the shape optimization problems (Sokolowski, 1993). 
Remark 4.8 For $T_{t}=I+t \Theta$, the associated velocity field takes the form $V=$ $\frac{\partial T_{t}}{\partial t} \circ T_{t}^{-1}=\Theta \circ(I+t \Theta)^{-1}$, whence the material derivative $\dot{V}(0)=\frac{d \Theta}{d t}=0$ in any direction $W$.

Hence for vector fiels $V(t, x)=\left[\Theta \circ(I+t \Theta)^{-1}\right](x), W(t, x)=\left[\Psi \circ(I+t \Psi)^{-1}\right](x)$, we have

$$
d^{2} J(\Omega ; V, W)=\mathfrak{d}^{2} J(\Omega ; V(0), W(0))=\mathfrak{d}^{2} J(\Omega ; \Theta, \Psi),
$$

and the second order Frechet derivative evaluated by the method of perturbation of identity is symmetric, hence

$$
\mathfrak{d}^{2} J(\Omega ; \Theta, \Psi)=\mathfrak{d}^{2} J(\Omega ; \Psi, \Theta) \quad \text { for all admissible vector fields } \Psi, \Theta
$$

Therefore, the second order derivative $\mathfrak{d}^{2} J(\Omega ; V(0), W(0))$ is symmetric with respect to directions $V(0), W(0)$ whenever the second order shape derivative $d^{2} J(\Omega ; V, W)$ exists.

Results on the second order differentiability of shape functionals were derived by several authors, eg. by N. Fujii, Z. Mróz and H. Petryk, J. Simon, P. Guillaume and M. Masmoudi.

\section{The first order shape sensitivity analysis}

The displacement derivative of solutions to (2.1) is given by the solutions to the following system.

Theorem 5.1 Assume we are given the displacement derivatives $\frac{\delta e_{t}}{\delta t}$ and $\frac{\delta \varphi_{t}}{\delta t}$.

The derivative $\vec{u}^{\prime}=\left.\frac{\delta \vec{u}_{t}}{\delta t}\right|_{t=0}$ of solution to equation (3.3) at $t=0$ is the unique solution to the equation

$$
\begin{gathered}
\text { find } \vec{u}^{\prime} \in \mathcal{H}=\left(H_{0}^{1}(\mathcal{O})\right)^{2} \times H_{0}^{2}(\mathcal{O}) \text { such that } \\
a\left(\vec{u}^{\prime}, \vec{v}\right)+\frac{\partial a_{t}}{\partial \varphi_{t}}(\vec{u}, \vec{v}){\frac{\delta \varphi_{t}}{\delta t}}_{\mid t=0}+\frac{\partial a_{t}}{\partial e_{t}}(\vec{u}, \vec{v}){\frac{\delta e_{t}}{\delta t}}_{\mid t=0}=\frac{\delta f_{t}}{\delta t}(\vec{v})_{\mid t=0} \\
\forall \vec{v} \in\left(H_{0}^{1}(\mathcal{O})\right)^{2} \times H_{0}^{2}(\mathcal{O})
\end{gathered}
$$

where the subsequent terms are given below and $\vec{u}$ denotes a solution to (2.1).

RR n 2995 
We have

$$
\frac{d}{d t}\left[f_{t}(\vec{v})\right]_{\mid t=0}=\int_{\mathcal{O}}\left[\left.\frac{\partial \vec{p}_{t}}{\partial t}\right|_{\mid t=0} \cdot \vec{v}+w_{t}{\frac{\partial \vec{p}_{t}}{\partial n}}_{\mid t=0} \cdot \vec{v}-2 w \kappa \vec{p}_{t \mid t=0} \cdot \vec{v}\right] d S .
$$

Here we use the following notation.

$$
\begin{aligned}
& \frac{\partial a_{t}}{\partial u_{t}}\left(\frac{\delta \vec{u}_{t}}{\delta t}, \vec{v}\right)=\int_{\mathcal{O}} e_{t} E_{t}^{\alpha \beta \iota \mu}\left\{\gamma_{t \alpha \beta}\left(\frac{\delta \vec{u}_{t}}{\delta t}\right) \gamma_{t \lambda \mu}(\vec{v})\right. \\
& \left.+\frac{e_{t}^{2}}{12} \bar{\rho}_{t \alpha \beta}\left(\frac{\delta \vec{u}_{t}}{\delta t}\right) \bar{\rho}_{t \lambda \mu}(\vec{v})\right\} d S_{t} \\
& \frac{\partial a_{t}}{\partial \varphi_{t}}\left(\vec{u}_{t}, \vec{v}\right) \frac{\delta \varphi_{t}}{\delta t} \\
& =\int_{\mathcal{O}} 2 e_{t} w_{t}\left\{\frac{E}{1+\nu}\left[a_{t}^{\alpha \lambda} b_{t}^{\beta \mu}+a_{t}^{\alpha \mu} b_{t}^{\beta \lambda}+\frac{\nu}{1+\nu}\left(a_{t}^{\alpha \beta} b_{t}^{\lambda \mu}+a_{t}^{\lambda \mu} b_{t}^{\alpha \beta}\right)\right]\right. \\
& \left.-\kappa_{t} E_{t}^{\alpha \beta \lambda \mu}\right\}\left\{\gamma_{t \alpha \beta}\left(\vec{u}_{t}\right) \gamma_{t \lambda \mu}(\vec{v})+\frac{e_{t}^{2}}{12} \bar{\rho}_{t \alpha \beta}\left(\vec{u}_{t}\right) \bar{\rho}_{t \lambda \mu}(\vec{v})\right\} \\
& -e_{t} E_{t}^{\alpha \beta \lambda \mu}\left\{\left[u_{t \iota} \frac{\delta}{\delta t}\left(\gamma_{t \alpha \beta}^{\iota}\right)+u_{t 3} \frac{\delta}{\delta t}\left(b_{t \alpha \beta}\right)\right] \gamma_{t \lambda \mu}(\vec{v})\right. \\
& +\gamma_{t \alpha \beta}\left(\vec{u}_{t}\right)\left[v_{\iota} \frac{\delta}{\delta t}\left(\Gamma_{t \lambda \mu}^{\iota}\right)+v_{3} \frac{\delta}{\delta t}\left(b_{t \lambda \mu}\right)\right] \\
& +\frac{e_{t}^{2}}{12}\left[u_{t 3, l} \frac{\delta}{\delta t}\left(\Gamma_{t \alpha \beta}^{\iota}\right)+u_{t 3} \frac{\delta}{\delta t}\left(b_{t \alpha}^{\omega} b_{t \omega \beta}\right)-u_{t \omega} \frac{\delta}{\delta t}\left(\left.b_{t \alpha}^{\omega}\right|_{\beta}\right)-u_{t \omega, \beta} \frac{\delta}{\delta t}\left(b_{t \alpha}^{\omega}\right)\right. \\
& \left.+u_{t \iota} \frac{\delta}{\delta t}\left(b_{t \alpha}^{\omega} \Gamma_{t \omega \beta}^{\iota}\right)-u_{t \omega, \alpha} \frac{\delta}{\delta t}\left(b_{t \beta}^{\omega}\right)+u_{t \iota} \frac{\delta}{\delta t}\left(b_{t \beta}^{\omega} \Gamma_{t \omega \alpha}^{\iota}\right)\right] \bar{\rho}_{t \lambda \mu}(\vec{v}) \\
& +\frac{e_{t}^{2}}{12} \bar{\rho}_{t \alpha \beta}\left(\vec{u}_{t}\right)\left[v_{3, \iota} \frac{\delta}{\delta t}\left(\Gamma_{t \lambda \mu}^{\iota}\right)+v_{3} \frac{\delta}{\delta t}\left(b_{t \lambda}^{\omega} b_{t \omega \mu}\right)-v_{\omega} \frac{\delta}{\delta t}\left(\left.b_{t \lambda}^{\omega}\right|_{\mu}\right)-v_{\omega, \mu} \frac{\delta}{\delta t}\left(b_{t \lambda}^{\omega}\right)\right. \\
& \left.\left.+v_{\iota} \frac{\delta}{\delta t}\left(b_{t \lambda}^{\omega} \Gamma_{t \omega \mu}^{\iota}\right)-v_{\omega, \lambda} \frac{\delta}{\delta t}\left(b_{t \mu}^{\omega}\right)+v_{\iota} \frac{\delta}{\delta t}\left(b_{t \mu}^{\omega} \Gamma_{t \omega \lambda}^{\iota}\right)\right]\right\} d S_{t} \\
& \frac{\partial a_{t}}{\partial e_{t}}\left(\vec{u}_{t}, \vec{v}\right) \frac{\delta e_{t}}{\delta t} \\
& =\int_{\mathcal{O}} E_{t}^{\alpha \beta \lambda \iota} \frac{\delta e_{t}}{\delta t}\left\{\gamma_{t \alpha \beta}\left(\vec{u}_{t}\right) \gamma_{t \lambda \iota}(\vec{v})+\frac{e_{t}^{2}}{4} \bar{\rho}_{t \alpha \beta}\left(\vec{u}_{t}\right) \bar{\rho}_{t \lambda \iota}(\vec{v})\right\} d S_{t} \cdot \\
& +k
\end{aligned}
$$

Remark 5.2 The form of displacement derivatives used in the above formulae can be obtained by direct computations. We refer the reader for such formulae to (Kosinski, 1986) and (Khludnev, Sokolowski, to appear).

Let $\mathfrak{J}\left(\varphi_{t}, e_{t} ; \vec{u}_{t}\right)$ be a functional that we are going to optimize. We assume that

$$
\mathfrak{J}\left(\varphi_{t}, e_{t} ; \vec{u}_{t}\right)=J\left(S_{t}\right), \text { where } S_{t}=\varphi_{t}(\mathcal{O})
$$


and $J\left(S_{t}\right)$ is a shape functional.

Put

$$
j(t)=\mathfrak{J}\left(\varphi_{t}, e_{t} ; \vec{u}_{t}\right)
$$

Under the assumption that data are sufficiently smooth and $\mathfrak{J}$ is differentiable, we can compute (Chenais, 1987)

$$
\begin{gathered}
\frac{d j}{d t}(0)=\lim _{t \rightarrow 0} \frac{j(t)-j(0)}{t} \\
=\left[\frac{\partial \mathfrak{J}}{\partial \varphi_{t}}\left(\varphi, e_{t} ; \vec{u}_{t}\right) \frac{\partial \varphi_{t}}{\partial t}+\frac{\partial \mathfrak{J}}{\partial e_{t}}\left(\varphi, e_{t} ; \vec{u}_{t}\right) \frac{\partial e_{t}}{\partial t}+\frac{\partial \mathcal{J}}{\partial u_{t}}\left(\varphi, e_{t} ; \vec{u}_{t}\right) \frac{\partial \vec{u}_{t}}{\partial t}\right]_{t=0} .
\end{gathered}
$$

Denote

$$
\frac{\delta j}{\delta t}(0)=\left[\frac{\partial \mathfrak{J}}{\partial \varphi_{t}}\left(\varphi, e_{t} ; \vec{u}_{t}\right) \frac{\delta \varphi_{t}}{\delta t}+\frac{\partial \mathfrak{J}}{\partial e_{t}}\left(\varphi, e_{t} ; \vec{u}_{t}\right) \frac{\delta e_{t}}{\delta t}+\frac{\partial \mathcal{J}}{\partial u_{t}}\left(\varphi, e_{t} ; \vec{u}_{t}\right) \frac{\delta \vec{u}_{t}}{\delta t}\right]_{t=0}
$$

By applying the structure theorem 4.1, it follows that,

$$
\frac{d j}{d t}(0)=\frac{\delta j}{\delta t}(0)
$$

Since, in general, we are not able to compute $\frac{\delta \vec{u}_{t}}{\delta t}$ for any $\frac{\delta \varphi_{t}}{\delta t}$, we introduce the adjoint state equation

Find $\vec{q} \in\left(H_{0}^{1}(\mathcal{O})\right)^{2} \times H_{0}^{2}(\mathcal{O})$ such that

$$
a(\vec{q}, \vec{v})=\left[\frac{\partial \mathcal{J}}{\partial u_{t}}\left(\varphi_{t}, e_{t} ; \vec{u}_{t}\right)\right]_{\mid t=0} \vec{v}, \quad \forall \vec{v} \in\left(H_{0}^{1}(\mathcal{O})\right)^{2} \times H_{0}^{2}(\mathcal{O})
$$

Clearly, equation (5.4) is uniquely solvable and expression (5.2) takes the form

$$
\begin{gathered}
\frac{d j}{d t}(0)=-\left[\frac{\partial a_{t}}{\partial u_{t}}\left(\vec{u}_{t}, \vec{q}\right) \frac{\delta \varphi_{t}}{\delta t}\right]_{\mid t=0}-\left[\frac{\partial a_{t}}{\partial e_{t}}\left(\vec{u}_{t}, \vec{q}\right) \frac{\delta e_{t}}{\delta t}\right]_{\mid t=0} \\
+\left[\frac{\delta f_{t}}{\delta t}(\vec{q})\right]_{\mid t=0}+\left[\frac{\partial \mathfrak{J}}{\partial \varphi_{t}}\left(\varphi_{t}, e_{t} ; \vec{u}_{t}\right) \frac{\delta \varphi_{t}}{\delta t}\right]_{\mid t=0}+\left[\frac{\partial \mathfrak{J}}{\partial e_{t}}\left(\varphi_{t}, e_{t} ; \vec{u}_{t}\right) \frac{\delta e_{t}}{\delta t}\right]_{\mid t=0},
\end{gathered}
$$

where the subsequent terms of the right-hand side can be obtained in the explicite form (Khludnev, Sokolowski, to appear). One should note that the right-hand side is actually a linear mapping with respect to $\left(w=\frac{\delta \varphi_{t}}{\delta t}, \varepsilon=\frac{\delta e_{t}}{\delta t}, t=0\right)$. 


\section{The second order shape sensitivity analysis}

For any $t \geq 0,(5.2)$ can be written as

$$
\frac{d j}{d t}(t)=\frac{\partial \mathfrak{J}}{\partial \varphi_{t}}\left(\varphi, e_{t} ; \vec{u}_{t}\right) \frac{\delta \varphi_{t}}{\delta t}+\frac{\partial \mathfrak{J}}{\partial e_{t}}\left(\varphi, e_{t} ; \vec{u}_{t}\right) \frac{\delta e_{t}}{\delta t}+\frac{\partial \mathcal{J}}{\partial u_{t}}\left(\varphi, e_{t} ; \vec{u}_{t}\right) \frac{\delta \vec{u}_{t}}{\delta t}
$$

and for any $t \geq 0$ we can define the following adjoint state equation.

Find $\vec{q}_{t} \in\left(H_{0}^{1}(\mathcal{O})\right)^{2} \times H_{0}^{2}(\mathcal{O})$ such that

$$
a_{t}\left(\vec{q}_{t}, \vec{v}\right)=\frac{\partial \mathcal{J}}{\partial u_{t}}\left(\varphi, e_{t} ; \vec{u}_{t}\right) \vec{v}, \quad \forall \vec{v} \in\left(H_{0}^{1}(\mathcal{O})\right)^{2} \times H_{0}^{2}(\mathcal{O})
$$

As in Theorem 4.1, we get the following result.

Theorem 6.1 The displacement derivative $\frac{\delta \vec{u}_{t}}{\delta t}$ of a solution to (3.3) is a unique solution to the following equation given $\left(\frac{\delta e_{t}}{\delta t}, \frac{\delta \varphi_{t}}{\delta t}\right)$, find $\frac{\delta \vec{u}_{t}}{\delta t} \in\left(H_{0}^{1}(\mathcal{O})\right)^{2} \times H_{0}^{2}(\mathcal{O})$ such that

$$
\begin{gathered}
a\left(\frac{\delta \vec{u}_{t}}{\delta t}, \vec{v}\right)+\frac{\partial a_{t}}{\partial \varphi_{t}}\left(\vec{u}_{t}, \vec{v}\right) \frac{\delta \varphi_{t}}{\delta t}+\frac{\partial a_{t}}{\partial e_{t}}\left(\vec{u}_{t}, \vec{v}\right) \frac{\delta e_{t}}{\delta t}=\frac{\delta f_{t}}{\delta t}(\vec{v}) \\
\forall \vec{v} \in\left(H_{0}^{1}(\mathcal{O})\right)^{2} \times H_{0}^{2}(\mathcal{O})
\end{gathered}
$$

where the subsequent terms are given in theorem 5.1

For $t=0$ we denote

$$
a\left(\vec{u}^{\prime}, \vec{v}\right)+\frac{\partial a_{t}}{\partial \varphi_{t}}(\vec{u}, \vec{v}){\frac{\delta \varphi_{t}}{\delta t}}_{\mid t=0}+\frac{\partial a_{t}}{\partial e_{t}}(\vec{u}, \vec{v}){\frac{\delta e_{t}}{\delta t}}_{\mid t=0}=\frac{\delta f_{t}}{\delta t}(\vec{v})_{\mid t=0}
$$

Furthermore, the displacement derivative $\frac{\delta \vec{q} t}{\delta t}$ of a solution to (6.2) is given as a unique solution to the equation

find $\frac{\delta \vec{q}_{t}}{\delta t} \in\left(H_{0}^{1}(S)\right)^{2} \times H_{0}^{2}(S)$ such that

$$
\begin{gathered}
a\left(\frac{\delta \vec{q}_{t}}{\delta t}, \vec{v}\right)+\frac{\partial a_{t}}{\partial \varphi_{t}}\left(\vec{q}_{t}, \vec{v}\right) \frac{\delta \varphi_{t}}{\delta t}+\frac{\partial a_{t}}{\partial e_{t}}\left(\vec{q}_{t}, \vec{v}\right) \frac{\delta e_{t}}{\delta t} \\
=\frac{\partial^{2} J}{\left(\partial u_{t}\right)^{2}}\left(\varphi_{t}, e_{t} ; \frac{\delta \vec{u}_{t}}{\delta t}\right) \vec{v}+\frac{\partial^{2} J}{\partial u_{t} \partial \varphi_{t}}\left(\varphi_{t}, e_{t} ; \vec{u}_{t}\right)\left(\vec{v}, \frac{\delta \varphi_{t}}{\delta t}\right) \\
+\frac{\partial^{2} J}{\partial u_{t} \partial e_{t}}\left(\varphi_{t}, e_{t} ; \vec{u}_{t}\right)\left(\vec{v}, \frac{\delta e_{t}}{\delta t}\right)
\end{gathered}
$$




$$
\forall \vec{v} \in\left(H_{0}^{1}(\mathcal{O})\right)^{2} \times H_{0}^{2}(\mathcal{O})
$$

Therefore, by relations $(6.1)-(6.3)$, we obtain

$$
\begin{gathered}
\frac{d j}{d t}(t)=\int_{\mathcal{O}} G_{t} d S_{t}=-\frac{\partial a_{t}}{\partial \varphi_{t}}\left(\vec{u}_{t}, \vec{q}_{t}\right) \frac{\delta \varphi_{t}}{\delta t}-\frac{\partial a_{t}}{\partial e_{t}}\left(\vec{u}_{t}, \vec{q}_{t}\right) \frac{\delta e_{t}}{\delta t} \\
+\frac{\delta f_{t}}{\delta t}\left(\vec{q}_{t}\right)+\frac{\partial \mathfrak{J}}{\partial \varphi_{t}}\left(\varphi, e_{t} ; \vec{u}_{t}\right) \frac{\delta \varphi_{t}}{\delta t}+\frac{\partial \mathfrak{J}}{\partial e_{t}}\left(\varphi, e_{t} ; \vec{u}_{t}\right) \frac{\delta e_{t}}{\delta t} .
\end{gathered}
$$

Using (5.1) it follows that

$$
\frac{d j}{d t}(t)=d J\left(S_{t} ; V(t)\right), \quad V(t)=\frac{\partial \varphi_{t}}{\partial t} \circ \varphi_{t}^{-1}
$$

therefore,

Corollary 6.2 The second order Eulerian derivative of shape functional (5.1) (see section 4 for the definition) is given by

$$
\mathfrak{D}^{2} J(S ; V(0), V(0))=\frac{d^{2} j}{d t^{2}}(0)=\int_{\mathcal{O}}\left[{\frac{\delta G_{t}}{\delta t}}_{\mid t=0}-2 w \kappa G\right] d S
$$

We obtain the form of the Shape Hessian using the latter formula.

Corollary 6.3 We have

$$
\begin{aligned}
& \mathfrak{d}^{2} J(S ; V(0), V(0))=-2 \int_{\mathcal{O}} w \kappa G d S \\
& +\frac{\partial^{2} J}{\left(\partial \varphi_{t}\right)^{2}}\left(\varphi, e_{t} ; \vec{u}_{t}\right)\left(\frac{\delta \varphi_{t}}{\delta t}\right)^{2}+2 \frac{\partial^{2} J}{\partial \varphi_{t} \partial e_{t}}\left(\varphi, e_{t} ; \vec{u}_{t}\right)\left(\frac{\delta \varphi_{t}}{\delta t}, \frac{\delta e_{t}}{\delta t}\right) \\
& +\frac{\partial^{2} J}{\partial \varphi_{t} \partial u_{t}}\left(\varphi, e_{t} ; \vec{u}_{t}\right)\left(\frac{\delta \varphi_{t}}{\delta t}, \frac{\delta \vec{u}_{t}}{\delta t}\right)+\frac{\partial^{2} J}{\left(\partial e_{t}\right)^{2}}\left(\varphi, e_{t} ; \vec{u}_{t}\right)\left(\frac{\delta e_{t}}{\delta t}\right)^{2} \\
& +\frac{\partial^{2} J}{\partial e_{t} \partial u_{t}}\left(\varphi, e_{t} ; \vec{u}_{t}\right)\left(\frac{\delta e_{t}}{\delta t}, \frac{\delta \vec{u}_{t}}{\delta t}\right)+\frac{d}{d t} f_{t}\left(\frac{\delta \vec{q}_{t}}{\delta t}\right) \\
& \left.-\frac{\partial^{2} a_{t}}{(} \partial \varphi_{t}\right)^{2}\left(\vec{u}_{t}, \vec{q}_{t}\right)\left(\frac{\delta \varphi_{t}}{\delta t}\right)-2 \frac{\partial^{2} a_{t}}{\partial \varphi_{t} \partial e_{t}}\left(\vec{u}_{t}, \vec{q}_{t}\right)\left(\frac{\delta \varphi_{t}}{\delta t}, \frac{\delta e_{t}}{\delta t}\right) \\
& -\frac{\partial a_{t}}{\partial \varphi_{t}}\left(\frac{\delta \vec{u}_{t}}{\delta t}, \vec{q}_{t}\right) \frac{\delta \varphi_{t}}{\delta t}-\frac{\partial a_{t}}{\partial \varphi_{t}}\left(\vec{u}_{t}, \frac{\delta \vec{q}_{t}}{\delta t}\right) \frac{\delta \varphi_{t}}{\delta t} \\
& -\frac{\partial^{2} a_{t}}{\left(\partial e_{t}\right)^{2}}\left(\vec{u}_{t}, \vec{q}_{t}\right)\left(\frac{\delta e_{t}}{\delta t}\right)^{2}-\frac{\partial a_{t}}{\partial e_{t}}\left(\frac{\delta \vec{u}_{t}}{\delta t}, \vec{q}_{t}\right) \frac{\delta e_{t}}{\delta t}-\frac{\partial a_{t}}{\partial e_{t}}\left(\vec{u}_{t}, \frac{\delta \vec{q}_{t}}{\delta t}\right) \frac{\delta e_{t}}{\delta t}
\end{aligned}
$$

RR n 2995 
Jan Sokolowski

\section{References}

[1] M. Bernadou and J.M. Boisserie, The Finite Element Method, in Thin Shell Thery: Application to Arch Dam Simulations, Birkhäuser, Boston, 1982.

[2] M. Bernadou and P.G. Ciarlet, Sur l'ellipticité du modèle linéaire de coques de W.T. Koiter, in Computing Methods in Applied Sciences and Engineering (R. Glowinski and J.L. Lions, Eds.), pp. 89-136, Lectures Notes in Economics and Mathematical Systems, 134, Springer Verlag, Berlin, 1976.

[3] M. Bernadou and B. Lalanne, On the approximation of thin shell by "B-spline and Finite Element Methods", in Innovative Numerical Methods in Engineering, Edited by R.P. Shaw, J. Périaux, A. Chaudouet, J. Wu, C. Marino and C.A. Brebbia, Springer Verlag, Berlin, pp. 585-592, 1986.

[4] M. Bernadou, F.J. Palma and B. Rousselet, Shape optimization of an elastic thin shell under various criteria, Structural Optimization 3, pp. 7-21.

[5] M. Bernadou, Finite Element Methods for Thin Shell Problems, John Wiley and Sons, London, 1995.

[6] M. Bernadou, P.G. Ciarlet and B. Miara, Existence theorems for two-dimensional linear shell theories, J. Elasticity, 34, pp. 111-138, 1994.

[7] D. Chenais Optimal design of midsurface of shells: differentiability proof and sensitivity computations, Appl. Math. Optim. 16, pp. 93-133, 1987.

[8] M. Delfour and J.P. Zolesio, Velocity method and Lagrangian formulation for the computation of the shape Hessian, SIAM J. Control and Optimization 29, pp. 1414-1442, 1991.

[9] G. Duvaut and J.L. Lions, Les Inéquations en Méchanique et en Physique, Dunod, Paris, 1972, English translation: Inequalities in Mechanics and Physics, Grundlehren der mathematischen Wissenschaften 219. Springer-Verlag, Berlin, 1976 .

[10] N. Fujii, Second order necessary conditions in a domain optimization problem, J.O.T.A. 65(2), pp. 223-244, 1990.

[11] A.M. Khludnev and J. Sokolowski, Modelling and Control in Solid Mechanics, International Series of Numerical Mathematics, Birkhäuser Verlag, to appear. 
[12] W.T. Koiter, On the foundations of the linear theory of thin elastic shells, Proc. Kon. Ned. Akad. B73, pp. 169-195, 1970.

[13] W. Kosinski, Introduction to field singularities and wave analysis, PWN - Ellis Horwood, Warsaw, 1985.

[14] J.L. Lions, Contrôle Optimal de Systèmes Gouvernés par des Equations aux Dérivées Partielles, Dunod, Paris, 1968, (English translation: Springer Verlag).

[15] A. Novruzi and J.R. Roche, Newton and Quasi-Newton methods in numerical computations of free surfaces in the electromagnetic shaping of liquid metals, Zeszyty Naukowe Politechniki Śląskiej, Seria: Mechanika, 116, Gliwice, pp. 149$160,1994$.

[16] H. Petryk and Z. Mroz, Time derivatives of integrals and functionals defined on varying volume and surface domains, Arch. Mech., 38, pp. 697-724, 1986.

[17] B. Rousselet, Shape design sensitivity from partial differential equations to implementation, Eng. Opt. 11, pp. 151-171, 1987.

[18] J. Sokolowski, Differential stability of control constrained optimal control problems for distributed parameter systems, In: Distributed Parameter Systems. Kappel, F. Kunisch, K. and Schappacher, W. (Eds.), Lecture Notes in Control and Information Sciences, 75, Springer Verlag, pp. 382-399, 1985.

[19] J. Sokolowski, Shape sensitivity analysis of boundary optimal control problems for parabolic systems, SIAM J. on Control and Optimization, 26, pp. 763-787, 1988 .

[20] J. Sokolowski, Stability of solutions to shape estimation problems, Mechanics of Structures and Machines 21(1), pp. 67-94, 1993.

[21] J. Sokolowski and J.-P. Zolesio, Introduction to Shape Optimization. Shape sensitivity analysis, Springer Verlag, New York, 1992.

RR n2995 
Unit`e de recherche INRIA Lorraine, Technop^ole de Nancy-Brabois, Campus scientifique, 615 rue du Jardin Botanique, BP 101, 54600 VILLERS LÈS NANCY

Unit'e de recherche INRIA Rennes, Irisa, Campus universitaire de Beaulieu, 35042 RENNES Cedex Unit 'e de recherche INRIA Rh`one-Alpes, 655, avenue de l'Europe, 38330 MONTBONNOT ST MARTIN Unit'e de recherche INRIA Rocquencourt, Domaine de Voluceau, Rocquencourt, BP 105, 78153 LE CHESNAY Cedex

Unit'e de recherche INRIA Sophia-Antipolis, 2004 route des Lucioles, BP 93, 06902 SOPHIA-ANTIPOLIS Cedex

Éditeur

INRIA, Domaine de Voluceau, Rocquencourt, BP 105, 78153 LE CHESNAY Cedex (France)

ISSN 0249-6399 